TecnoHumanismo. Revista Científica

Setiembre - Noviembre 2021

Volumen 1 / No.3

ISSN: $2710-2394$

pp. $266-275$

https://doi.org/10.53673/th.v1i12.82

https://tecnohumanismo.online

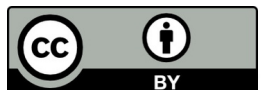

\title{
Enseñanza aprendizaje virtual en tiempos de pandemia en una Universidad Nacional
}

Teaching virtual learning in times of pandemic in a National University

Ensino de aprendizagem virtual em tempos de pandemia em uma Universidade Nacional

\section{ARTÍCULO GENERAL}

Jorge Antonio Acosta Piscoya

jacosta@unprg.edu.pe

https://orcid.org/0000-0002-2280-2974

Universidad Nacional Pedro Ruiz Gallo

Lambayeque-Perú

\author{
Débora Esther Mejía Pacheco \\ dmejia@unprg.edu.pe \\ https://orcid.org/0000-0003-0543-1021 \\ Universidad Nacional Pedro Ruiz Gallo \\ Lambayeque-Perú
}

Recibido 07 de Abril 2021 | Arbitrado y aceptado 19 de Junio 2021 | Publicado en 03 Setiembre 2021

\section{RESUMEN}

El Objetivo de la presente investigación es realizar un análisis de la enseñanza aprendizaje de las clases virtuales a nivel universitario en tiempos de pandemia, como consecuencia del mortal virus sars cov 2 o covid 19 que viene azotando al mundo, para lo cual se aplicó un instrumento de medición de 13 ítems en escala de Likert con cinco alternativas que varía desde totalmente en desacuerdo (1) hasta totalmente de Acuerdo (5) a los estudiantes de la Facultad de Ciencias Físicas y Matemáticas (FACFyM) de la Universidad Nacional Pedro Ruiz Gallo (UNPRG) de Lambayeque, en este estudio participaron 120 estudiantes de las diferentes carreras profesionales que conforman la FACFyM, el instrumento presento una fiabilidad del 0.894 , el $74,2 \%$ de los participantes son del sexo Masculino, así mismo el $65,8 \%$ manifiestan haber tenido dificultad con la señal de Internet, y el $16,7 \%$ señalaron no tener una buen receptor (Laptop, Table, celular, etc.), el ítems 12 es el más valorada que consideran que una de las ventajas de las clases virtuales es la disponibilidad de la información en internet y la menos valorada son el ítems 1 y 13 relacionadas con la satisfacción de la enseñanza aprendizaje virtual, los estudiantes de la escuela profesional de ingeniería en computación e informática son los que más valoran las clases virtuales y los que menos lo valoran son los estudiantes de la escuela profesional de ingeniería electrónica y Matemáticas, el KMO obtenido es 0,901 , la enseñanza virtual se puede explicar en dos factores con una varianza total explicada del $61,161 \%$, el factor uno agrupa las bondades que ofrece la virtualidad y el factor dos las deficiencias.

Palabra Clave: Enseñanza aprendizaje de las clases virtuales.

\section{ABSTRACT}

The objective of this research is to carry out an analysis of the teaching-learning of virtual classes at the university level in times of pandemic, as a consequence of the deadly virus sars cov 2 or covid 19 that has been hitting the world, for which an instrument was applied measurement of 13 items on a Likert scale with five alternatives that varies from totally disagree (1) to totally agree (5) to students of the Faculty of Physical and Mathematical Sciences (FACFyM) of the Pedro Ruiz Gallo National University ( UNPRG) of Lambayeque, 120 students from the different professional careers that make up the FACFyM participated in this study, the instrument presented a reliability of $0.894,74.2 \%$ of the participants are male, likewise $65.8 \%$ state having had difficulty with the Internet signal, and $16.7 \%$ indicated not having a good receiver (Laptop, Table, cell phone, etc.), item 12 is the most valued that they consider to be ue one of the advantages of virtual classes is the availability of information on the internet and the least valued are items 1 and 13 related to the satisfaction of virtual learning teaching, students of the professional school of computer engineering and informatics are Those who most value virtual classes and those who least value it are students from the professional school of electronic engineering and Mathematics, the KMO obtained is 0.901 , virtual teaching can be explained in two factors with a total explained variance of $61.161 \%$, factor one groups together the benefits offered by virtuality and factor two the deficiencies. Key Word: Teaching and learning of virtual classes.

\section{RESUMO}

O objetivo desta pesquisa é realizar uma análise do ensino-aprendizagem de aulas virtuais de nível universitário em tempos de pandemia, em decorrência do vírus mortal sars cov 2 ou covid 19 que vem atingindo o mundo, pelo qual foi aplicado um instrumento de medida de 13 itens em escala Likert com cinco alternativas que variam de discordo totalmente (1) a concordo totalmente (5) para os alunos da Faculdade de Ciências Físicas e Matemáticas (FACFyM) da Universidade Nacional Pedro Ruiz Gallo (UNPRG) de Lambayeque, participaram deste estudo 120 alunos das diferentes carreiras profissionais que compõem a FACFyM, o instrumento apresentou confiabilidade de 0,894, 74,2\% dos participantes são do sexo masculino, da mesma forma $65,8 \%$ afirmam ter tido dificuldade com o sinal da Internet, e $16,7 \%$ indicaram não ter um bom receptor (Laptop, Mesa, celular etc.), o item 12 é o mais valorizado que consideram ser ue uma das vantagens das aulas virtuais é a disponibilidade de informações na internet e os menos valorizados são os itens 1 e 13 relacionados à satisfação do ensino aprendizagem virtual, os alunos da escola profissional de engenharia da computação e informática são os que mais valorizam o virtual turmas e quem menos valoriza são os alunos da escola profissionalizante de engenharia eletrônica e matemática, o KMO obtido é de 0,901 , o ensino virtual pode ser explicado em dois fatores com uma variância total explicada de $61,161 \%$, o fator um agrupa os benefícios oferecidos pela virtualidade e fator dois as deficiências.

Palavra-chave: Ensino e aprendizagem de aulas virtuais. 


\section{Introducción}

El 11 de Marzo del 2020, a cinco días después de haberse detectado en Perú el primer caso positivo de COVID 19, el ministerio de Educación decide postergar el inicio de las clases en todas las escuelas publicas y privadas del país, esta misma medida se adopto para todas las universidades publicas y privadas del país. Wikipedia (2020)

Este hecho obligo a plantear otras formas de enseñanza aprendizaje adoptando la forma virtual, cada una de las instituciones tuvieron que crear sus respectivas plataformas virtuales, y capacitar a los docentes y alumnos en el uso de las TIC, algunas optaron por usar Zoom, otros Google meet, entre otras. Estas plataformas de video conferencia que permitieron continuar con el proceso de enseñanza aprendizaje, tienen sus ventajas y desventajas las cuales se trataron en el presente estudio.

Según Asmar (2002) citado por Alvarez (2002), En las sociedades modernas, las nuevas tecnologías de la información y la comunicación, adquieren un significado preponderante. La abundancia de información es infinita e imposible de ser asimilada, la complejidad del conocimiento es cada vez más grande, el cambio vertiginoso en los aspectos de la sociedad vuelve caducos los conocimientos y hábitos con gran celeridad, la educación virtual, surge como una necesidad de los tiempos modernos, donde el estudiante debe capacitarse en forma permanente, para lo cual requiere aprender a regular su propio ritmo de aprendizaje conciliando su tiempo de trabajo, de estudio, de socialización, de diversión y recreación, así como seleccionando por sí mismo las temáticas e información de su interés, de acuerdo con su propia necesidad, utilizando los diferentes medios de auto instrucción y comunicación que ofrece el mundo moderno.

BID (2020). Entre los aportes de la segunda reunión del dialogo virtual con rectores de universidades lideres de América latina, consideran que en el caso de la acreditación de los saberes del estudiante, la dinámica pedagógica de la educación virtual varia de la presencial, evidenciando que en algunos casos los estudiantes han sido sobrecargados debido al desconocimiento pedagógico virtual del docente, muchos estudiantes en la región tienen dificultades para acceder a computadoras o no tienen conectividad a su disposición, lo cual resulta en un incremento de la tasa de abandono de la aulas, muchos estudiantes viven en ambientes poco favorables para poder adaptarse a los formatos virtuales, considerando las condiciones de su hogar, la 
disposición de red y el debido acceso a las tecnologías requeridas. Así mismo Por medio de webinars y tutoriales se han desplegado esfuerzos permanentes para ayudar a los profesores a adaptarse a la teleeducación.

Bravo, F. (2021). En su investigación Educación virtual en la universidad en tiempos de covid-19, en una universidad del Ecuador donde participaron 176 estudiantes, manifiesta que la educación virtual debe continuar y debe ser mejorado estos procesos para el futuro, entre sus resultados encontrados hace notar que no todos los estudiantes cuentan con un dispositivo propio para escuchar sus clases virtuales, el $43 \%$ lo hace desde un teléfono, el 39\% manifiesta que la señal de internet es deficiente.

\section{Métodos y materiales}

La presente investigación es descriptiva se aplicó una encuesta virtual preparada en Google drive, la cual consistió en una primera parte informativa y en una segunda parte un conjunto de 13 interrogantes en escala de LIKERT que deberían ser valoradas desde Totalmente en Desacuerdo hasta Totalmente de acuerdo, se creó una base de datos, usando hoja de cálculo de Excel con apoyo de un software estadístico, primero se realizó un análisis diagnostico utilizando la Estadística descriptiva e inferencial, así mismo el análisis factorial exploratorio con rotación varimax.

\section{Resultados}

Dentro de los resultados encontrados al realizar el análisis exploratorio de la enseñanza y aprendizaje virtual en tiempos de pandemia en una universidad nacional, tenemos

Tabla 1.

Alumnos de la FACFyM por sexo según escuela profesional.

\begin{tabular}{|c|c|c|c|c|c|c|}
\hline \multirow{3}{*}{$\begin{array}{l}\text { ESCUELA } \\
\text { PROFESIONAL } \\
\end{array}$} & \multicolumn{4}{|c|}{ SEXO DEL ENCUESTADO } & & \\
\hline & \multicolumn{2}{|c|}{ MASCULINO } & \multicolumn{2}{|c|}{ FEMENINO } & \multicolumn{2}{|c|}{ Total } \\
\hline & $\mathrm{N}^{\mathrm{o}}$ & $\%$ & $\mathrm{~N}^{\mathrm{o}}$ & $\%$ & $\mathrm{~N}^{\mathrm{o}}$ & $\%$ \\
\hline Estadística & 10 & 8,3 & 15 & 12,5 & 25 & 20,8 \\
\hline Física & 4 & 3,3 & 1 & 0,8 & 5 & 4,2 \\
\hline Ing. Computación & 26 & 21,7 & 8 & 6,7 & 34 & 28,3 \\
\hline Ing. Electrónica & 37 & 30,8 & 4 & 3,3 & 41 & 34,2 \\
\hline
\end{tabular}




\begin{tabular}{lcc|cc|cc} 
'Matemáticas & 12 & 10,0 & 3 & 2,5 & 15 & 12,5 \\
Total & 89 & 74,2 & 31 & 25,8 & 120 & 100,0 \\
\hline \hline
\end{tabular}

Nota: Base de encuesta educación virtual.

Los resultados que se muestran en la tabla 1, en el estudio participaron 120 estudiantes de las diferentes escuelas profesionales el 74,2\% son hombres, el $34.2 \%$ pertenecen a la escuela profesional de ingeniería electrónica, el 28,3\% a la escuela de ingeniería de computación e informática, el 20,8\% a la escuela de Estadística, 12,5\% a la escuela profesional de Matemáticas y solo el 4,2\% a la escuela profesional de Física.

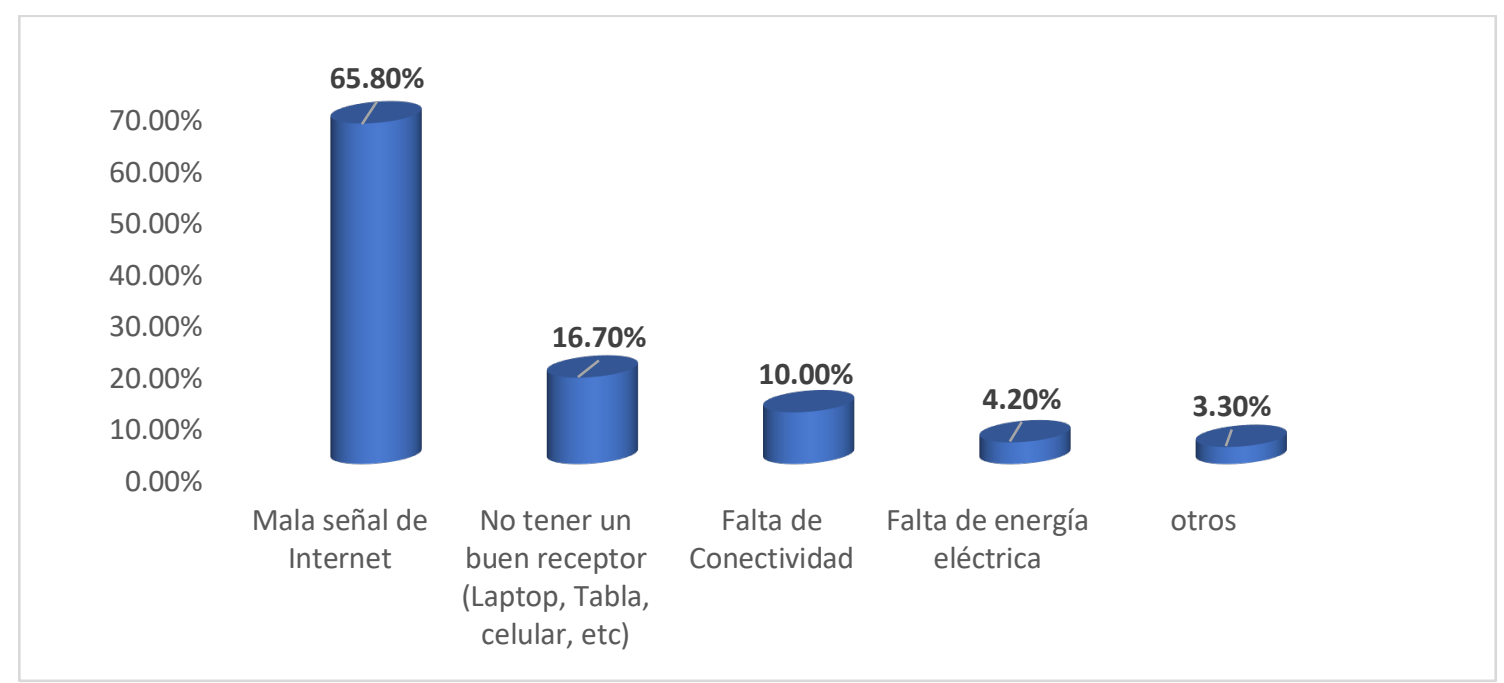

Figura 1. Dificultad para educación virtual.

Los resultados que se muestran en la figura 1, permitió determinar que el 65,8\% de los estudiantes manifestaron que la mayor dificultad que han tenido para desarrollar sus clases virtuales es la mala señal de internet, el 16,7\% señalaron también de no contar con un buen receptor (Laptop, Table, celular, etc.), el 10\% la falta de conectividad por el lugar donde se encontraban recibiendo su clase, el $4.2 \%$ por la falta de energía eléctrica y solo el 3.3\% señalan haber tenido otras dificultades para desarrollar sus clases virtuales, entre las que manifestaron no contar un ambiente adecuado. 
Tabla 2.

Análisis descriptivo de los ítems para evaluar la enseñanza aprendizaje de la educación virtual.

\begin{tabular}{|c|c|c|c|c|}
\hline & $\mathrm{N}^{\mathrm{o}}$ & $\mathrm{M}(\mathrm{x})$ & D.E & C.V $(\%)$ \\
\hline I.1. Te sientes conforme con las clases virtuales. & 120 & 3,13 & 1,206 & 38,5 \\
\hline $\begin{array}{l}\text { I.2. El docente es eficiente en el desarrollo de las } \\
\text { clases virtuales. }\end{array}$ & 120 & 3,55 & ,986 & 27,8 \\
\hline $\begin{array}{l}\text { I.3. Las estrategias utilizadas por los docentes } \\
\text { permiten cumplir con las competencias } \\
\text { planteadas en el sílabo. }\end{array}$ & 120 & 3,49 & ,987 & 28,3 \\
\hline $\begin{array}{l}\text { I.4. El docente se presenta adecuadamente vestido a } \\
\text { las clases. }\end{array}$ & 120 & 3,60 & 1,024 & 28,4 \\
\hline $\begin{array}{l}\text { I.5. Consideras que el aula virtual te permite } \\
\text { retroalimentar tu aprendizaje. }\end{array}$ & 120 & 3,33 & 1,116 & 33,5 \\
\hline $\begin{array}{l}\text { I.6. Consideras que el aula Google meet es } \\
\text { adecuada para el aprendizaje. }\end{array}$ & 120 & 3,48 & 1,115 & 32,0 \\
\hline $\begin{array}{l}\text { I.7. Consideras que la falta de conocimiento por } \\
\text { parte de algunos docentes en el uso de las TIC } \\
\text { no permite el desarrollo adecuado de la clase. }\end{array}$ & 120 & 3,33 & 1,038 & 31.2 \\
\hline $\begin{array}{l}\text { I.8. Consideras que cuando se vuelva a las clases } \\
\text { presenciales, el desarrollo de los cursos debe } \\
\text { conservar algunas horas virtuales. }\end{array}$ & 120 & 3,48 & 1,283 & 36,9 \\
\hline $\begin{array}{l}\text { I.9. Consideras que los trámites administrativos } \\
\text { virtuales son más eficientes. }\end{array}$ & 120 & 3,18 & 1,301 & 40,9 \\
\hline $\begin{array}{l}\text { I.10. Consideras que el aprendizaje virtual es } \\
\text { bueno. }\end{array}$ & 120 & 3,15 & 1,128 & 35,8 \\
\hline $\begin{array}{l}\text { I.11. Consideras que es muy poco lo que uno } \\
\text { aprende en las clases virtuales. }\end{array}$ & 120 & 3,34 & 1,213 & 36,3 \\
\hline $\begin{array}{l}\text { I.12. consideras que una de las ventajas de las } \\
\text { clases virtuales es la disponibilidad de la } \\
\text { información en internet. }\end{array}$ & 120 & 3,88 & 1,142 & 29,4 \\
\hline $\begin{array}{l}\text { I.13. Te encuentras satisfecho con la enseñanza } \\
\text { aprendizaje virtual. }\end{array}$ & 120 & 3,13 & 1,149 & 36,7 \\
\hline
\end{tabular}

Nota: Base de encuesta educación virtual.

Los resultados que se muestran en la tabla 2, nos permitió determinar que el ítem más valorado es el I.12 el estudiante considera que una de las ventajas de las clases virtuales es la disponibilidad de la información en internet, y el ítem menso valorado corresponde al I.1 y I13. Que tiene que ver con la satisfacción de las clases virtuales, así 
mismo el I.9 presenta mayor heterogeneidad $(\mathrm{CV}=40.9 \%)$ en las respuestas, este ítem esta relacionando con la atención administrativa, el I.2 es la más homogénea $(\mathrm{CV}=27.8 \%)$ en las respuestas vertidas por los estudiantes que esta relacionado con la eficiencia del docente en el desarrollo de las clases virtuales.

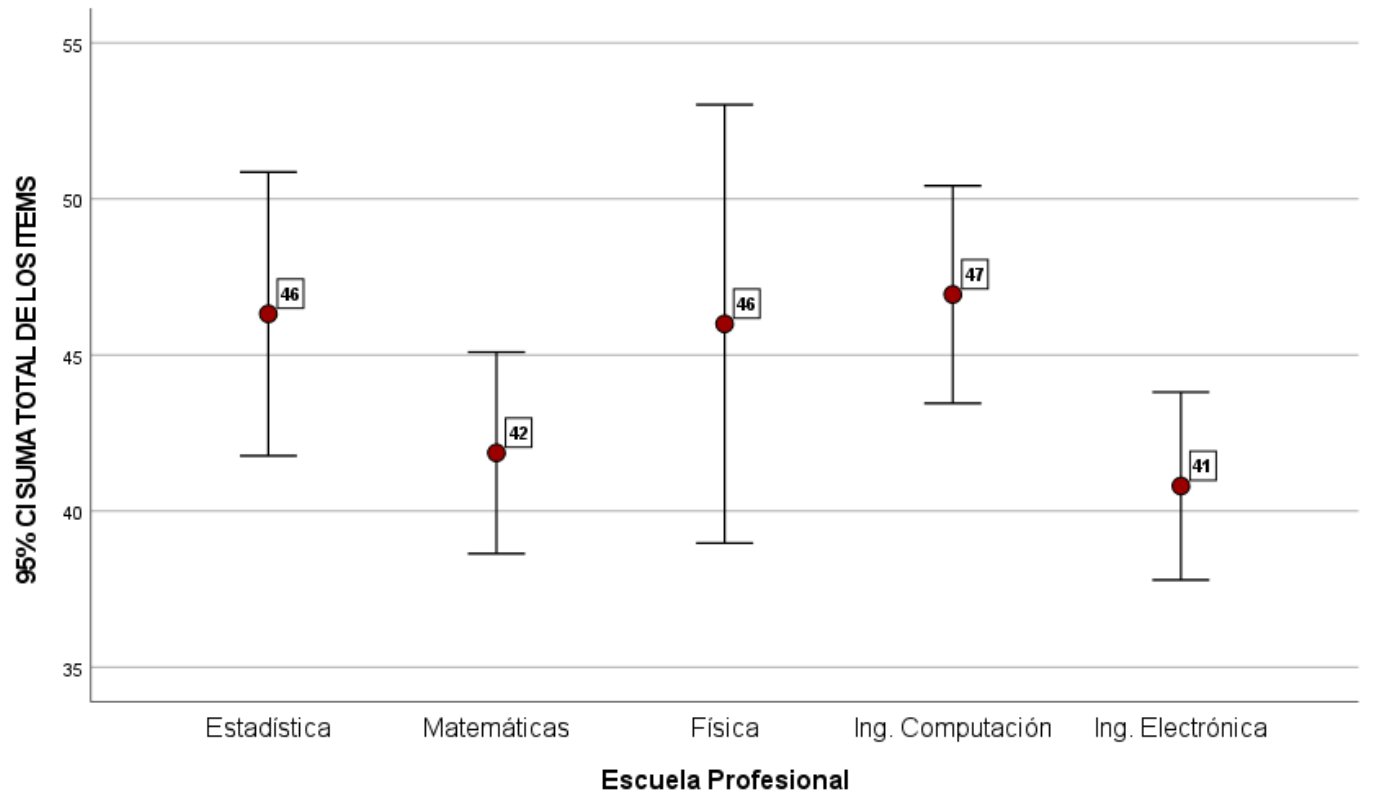

Figura 2. Valoración de los ítems

La Figura 2. Nos permite visualizar que en promedio los estudiantes de la escuela profesional de ingeniería en computación e informática son los que más valoran las clases virtuales y los que menos lo valoran son los estudiantes de ingeniería electrónica.

Tabla 3.

Varianza total explicada de la enseñanza aprendizaje de la educación virtual.

\begin{tabular}{|c|c|c|c|c|c|c|c|c|c|}
\hline \multirow[b]{2}{*}{ Componente } & \multicolumn{3}{|c|}{ Autovalores iniciales } & \multicolumn{3}{|c|}{$\begin{array}{c}\text { Sumas de cargas al cuadrado } \\
\text { de la extracción }\end{array}$} & \multicolumn{3}{|c|}{$\begin{array}{c}\text { Sumas de cargas al cuadrado } \\
\text { de la rotación }\end{array}$} \\
\hline & Total & $\begin{array}{c}\% \text { de } \\
\text { varianza } \\
\end{array}$ & $\begin{array}{c}\% \\
\text { acumulado } \\
\end{array}$ & Total & $\begin{array}{c}\% \text { de } \\
\text { varianza } \\
\end{array}$ & $\begin{array}{c}\% \\
\text { acumulado } \\
\end{array}$ & Total & $\begin{array}{c}\% \text { de } \\
\text { varianza } \\
\end{array}$ & $\begin{array}{c}\% \\
\text { acumulado } \\
\end{array}$ \\
\hline 1 & 6.433 & 49.487 & 49.487 & 6.433 & 49.487 & 49.487 & 6.212 & 47.783 & 47.783 \\
\hline 2 & 1.518 & 11.674 & 61.161 & 1.518 & 11.674 & 61.161 & 1.739 & 13.378 & 61.161 \\
\hline 3 & .954 & 7.340 & 68.501 & & & & & & \\
\hline$\cdots$ & $\cdots$ & $\cdots \cdots$ & $\ldots \ldots$ & & & & & & \\
\hline 12 & .151 & 1.161 & 98.903 & & & & & & \\
\hline 13 & .143 & 1.097 & 100.000 & & & & & & \\
\hline
\end{tabular}


La tabla 3 permitió determinar que la enseñanza aprendizaje de la educación se puede explicar en dos factores con una varianza explicada del $61,161 \%$. Los ítems que conforman cada uno de los factores se puede visualizar en la figura 4.

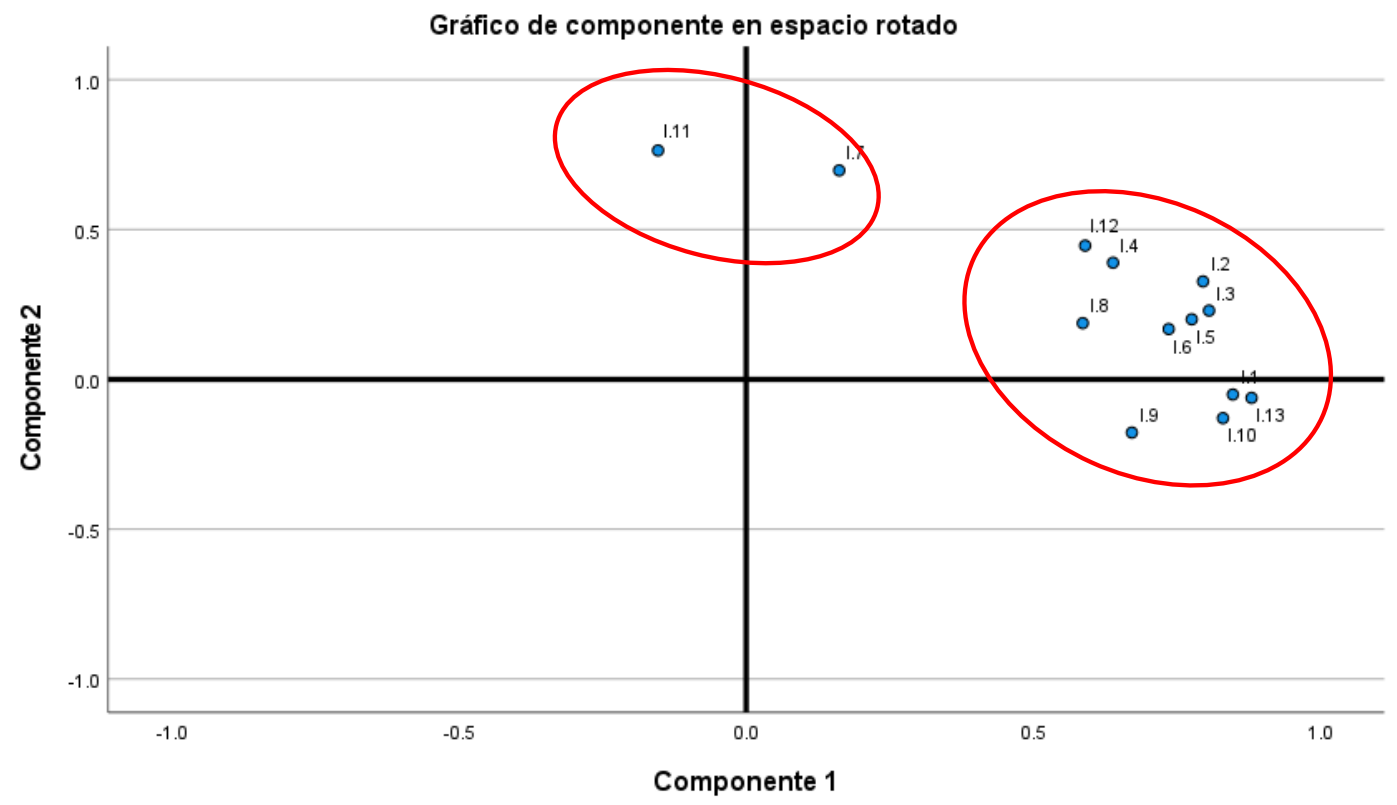

Figura 4. Factores rotados

Tabla 4.

Matriz de componentes rotados.

\begin{tabular}{lc}
\hline \hline & Componente \\
\cline { 2 - 2 } & 1 \\
\hline I.13. Te encuentras satisfecho con la enseñanza aprendizaje virtual. & $\mathbf{. 8 8 0}$ \\
I.1. Te sientes conforme con las clases virtuales. & $\mathbf{8 4 8}$ \\
I.10. Consideras que el aprendizaje virtual es bueno. & $\mathbf{. 8 3 0}$ \\
I.3. Las estrategias utilizadas por los docentes permiten cumplir con las & $\mathbf{. 8 0 6}$ \\
competencias planteadas en el sílabo. & $\mathbf{. 7 9 6}$ \\
I.2. El docente es eficiente en el desarrollo de las clases virtuales. & $\mathbf{. 7 7 6}$ \\
I.5. Consideras que el aula virtual te permite retroalimentar tu aprendizaje. & $\mathbf{. 7 3 6}$ \\
I.6. Consideras que el aula Google meet es adecuada para el aprendizaje. & $\mathbf{. 6 7 2}$ \\
I.9. Consideras que los trámites administrativos virtuales son más eficientes. & $\mathbf{. 6 3 9}$ \\
I.4. El docente se presenta adecuadamente vestido a las clases. & $\mathbf{. 5 9 0}$ \\
I.12. consideras que una de las ventajas de las clases virtuales es la & \\
disponibilidad de la información en internet. & $\mathbf{5 8 6}$
\end{tabular}

I.11. Consideras que es muy poco lo que uno aprende en las clases virtuales. 

uso de las TIC no permite el desarrollo adecuado de la clase.

Nota: Base de encuesta educación virtual.

Los resultados que se muestran en la tabla 4 permitió determinar que el factor uno conformado por 11 ítems agrupo a los que están relacionados con las bondades que ofrece el desarrollo de las clases virtuales y el factor dos conformados por dos ítems que consideran las deficiencias de la enseñanza aprendizaje de las clases virtuales.

\section{Discusion}

- Los resultados encontrados en la presente investigación son similares a los manifestados por los rectores de las universidades líderes de América Latina y de los encontrados por Bravo, donde la principal dificultad es la mala señal de internet debido a la ubicación donde reciben sus clases virtuales los estudiantes.

- El I.8. donde se le consulta al estudiante si estaría de acuerdo que cuando se vuelva a las clases presenciales, el desarrollo de los cursos debe conservar algunas horas virtuales, se mostró indiferente, esto debido a las dificultades que viene teniendo para recibir sus clases virtuales, esto no es solamente un problema del estudiante también involucra al docente como lo manifiesta los rectores de las universidades lideres de América latina.

- Aun no existen trabajos de la enseñanza aprendizaje virtual donde se aplique el análisis factorial para tratar de explicar este proceso, en la presente investigación se determinaron dos factores con una varianza explicada del $61,161 \%$, talvez no es la esperada, pero si suficiente para tratarla de entender el proceso de enseñanza aprendizaje virtual.

\section{Conclusiones}

- Los estudiantes de la escuela profesional de ingeniería en computación e informática son los que mas valoran las clases virtuales y los que menos la valoran son los estudiantes de la escuela profesional de ingeniería electrónica.

- $\quad$ El proceso de enseñanza aprendizaje virtual se puede explicar con dos factores el primero agrupa los once ítems relacionados con la bondad que brinda la virtualidad y el segundo dos ítems que muestran las deficiencias del proceso enseñanza aprendizaje de las clases virtuales. 


\section{Bibliogafia}

Álvarez, O y Zapata, D. (2002). La enseñanza virtual en la educación superior. https://www.fumc.edu.co/wp-content/uploads/resoluciones/arc 914.pdf

BID (2020). La educación superior en tiempos de covid-19.

https://publications.iadb.org/publications/spanish/document/La-educacionsuperior-en-tiempos-de-COVID-19-Aportes-de-la-Segunda-Reunion-delDi\%C3\%A1logo-Virtual-con-Rectores-de-Universidades-Lideres-de-AmericaLatina.pdf

Bravo, F. (2021). Educación Virtual en la Universidad en tiempos de covid-19. https://www.espirituemprendedortes.com/index.php/revista/article/view/238/311

Wikipedia (2020). Pandemia del covid-19 en Perú.

https://es.wikipedia.org/wiki/Pandemia de COVID-

19 en Per $\% \mathrm{C} 3 \% \mathrm{BA \# Educaci} \% \mathrm{C} 3 \% \mathrm{~B} 3 \mathrm{n}$ 